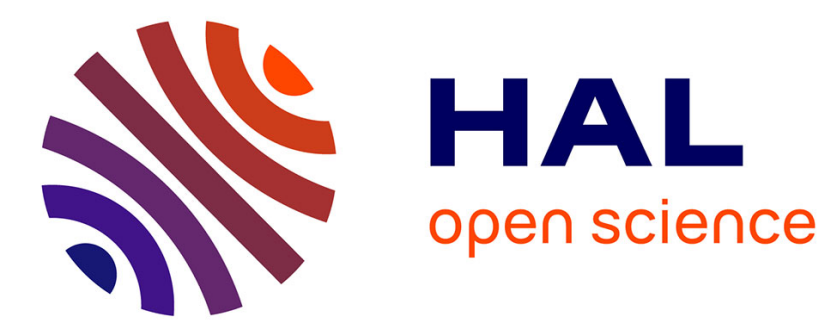

\title{
Avant-propos. Des enceintes en terre anhistoriques à Google Earth \\ Olivier Buchsenschutz
}

\section{To cite this version:}

Olivier Buchsenschutz. Avant-propos. Des enceintes en terre anhistoriques à Google Earth. Archimède : archéologie et histoire ancienne, 2016, 3, pp.2-7. 10.47245/archimede.0003.ds1.02 . hal01536220

\section{HAL Id: hal-01536220 \\ https://hal.science/hal-01536220}

Submitted on 10 Jun 2017

HAL is a multi-disciplinary open access archive for the deposit and dissemination of scientific research documents, whether they are published or not. The documents may come from teaching and research institutions in France or abroad, or from public or private research centers.
L'archive ouverte pluridisciplinaire HAL, est destinée au dépôt et à la diffusion de documents scientifiques de niveau recherche, publiés ou non, émanant des établissements d'enseignement et de recherche français ou étrangers, des laboratoires publics ou privés.

\section{(1)(1) $\$(0)$}

Distributed under a Creative Commons Attribution - NonCommercial - ShareAlikel 4.0 


\section{ARCHIMĖDE}

DOSSIER THÉMATIQUE : DES FOSSÉS ET DES REMPARTS. ENCEINTES ET SITES FORTIFIÉS DU RHIN SUPÉRIEUR ENTRE PROTOHISTOIRE ET MOYEN ÂGE

2 Olivier BUCHSENSCHUTZ

Avant-propos. Des enceintes en terre anhistoriques à Google Earth

8 Lizzie SCHOLTUS

Histoire de la recherche dans le bassin de Saint-Dié-des-Vosges

20 Maxime WALTER

Les sites de hauteur du massif vosgien. Actualisation des données et modalités d'implantation

37 Jean-Jacques SCHWIEN

Chateaux et enceintes des Vosges du Nord. Topographie et longue durée

49 Anne-Marie ADAM

La palissade dans tous ses états : I'enclos du Britzgyberg (Illfurth, Haut-Rhin) et autres aménagements palissadés dans les habitats du premier âge du Fer

60 Clément FÉLIU

L'enceinte inférieure du Frankenbourg (67) et les remparts à poteaux frontaux de la fin de l'âge du Fer dans l'espace du Rhin supérieur. Pour une révision de la typologie des Pfostenschlitzmauern

74 Jacky $\mathrm{KOCH}$ et Thomas FISCHBACH

Enceintes de hauteur en pierres et formes « primitives » de châteaux ? L'exemple du Bernstein

87 Adrien VUILLEMIN

Les enceintes urbaines en moyenne Alsace (1200-1850)

102 Jean-François PININGRE

Les enceintes de l'âge du Bronze et du premier âge du Fer en Franche-Comté. Un bilan des recherches

124 Clément FÉLIU et Jean-Jacques SCHWIEN

Conclusion. Nouvelles perspectives sur les enceintes du Rhin supérieur

\section{ACTUALITÉ DE LA RECHERCHE : ARCHÉOLOGIE DES RÉSEAUX}

\section{Claire CAMBERLEIN}

Les réseaux en archéologie : approche historiographique et interdisciplinaire

135 Thomas HUTIN

Lieux d'échanges et espaces publics en Gaule à La Tène finale

150 Streeve GENTNER

Économie du fer et voies de communication, de l'abattage du minerai à la distribution

du métal : I'exemple du nord de la Forêt-Noire au Ve siècle av. J.-C.

169 Loup BERNARD et Rémy WASSONG

Du Danemark au Fossé rhénan. Un siècle d'analyse des voies de communications protohistoriques : évolution des méthodes et mise en commun des données

184 Steeve GENTNER et Rémy WASSONG

Conclusion. L'archéologie des réseaux : une thématique aux multiples facettes

\section{VARIA}

187 Fábio VERGARA CERQUEIRA

To march in phalanx, to jump with weights, to tread the grapes, to knead the bread. What is the aulos for?

206 Hermann AMON

Les supra-commandements comme solution à la crise militaire du IIIe siècle de l'Empire romain sous Philippe I'Arabe et Gallien

218 Martina BONO

Il processo di Cremuzio Cordo in Dio LVII, 24, 2-4

\section{LA CHRONIQUE D'ARCHIMÈDE}

228 Frédéric COLIN (éd.)

La Chronique d'Archimède. Bilan des activités scientifiques 2015-2016 de I'unité mixte de recherche 7044 


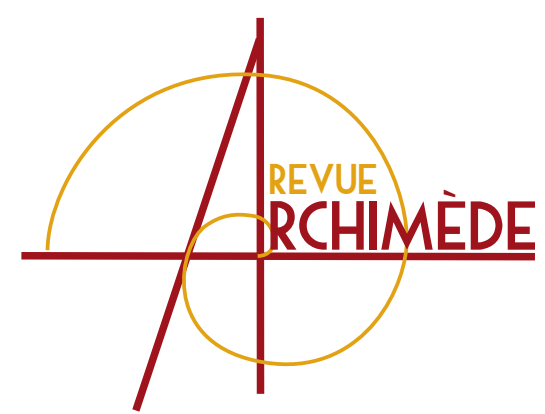

\section{AVANT-PROPOS. DES ENCEINTES EN TERRE ANHISTORIQUES À GOOGLE EARTH}

Olivier BUCHSENSCHUTZ

Directeur de recherche émérite

CNRS

AOROC UMR 8546

olivier.buchsenschutz@ens.fr

Cette introduction dresse un rapide tableau historique des recherches sur les enceintes dont les prémices remontent au XIX ${ }^{e}$ siècle. Les différentes méthodes mises en œuvres ont également passées en revue. L'intérêt des études diachroniques
This introduction provides a brief historical overview of research on hillforts, whose beginnings date back to the $19^{\text {th }}$ century. The various methods used are reviewed. Finally, the value of diachronic studies on fortifications is highlighted.
KeYWords

Hillforts, methods, maps, lidar. 
En associant dans la même réflexion la Protohistoire, I'Antiquité et le Moyen Âge, les organisateurs du colloque renouent heureusement avec une ancienne tradition. L'enquête de la Société Préhistorique Française (SPF) dans le premier quart du XXe $\mathbf{s}$. parlait, pour souligner la difficulté de les dater, d'enceintes « anhistoriques», et s'intéressait essentiellement aux fortifications en terre. Cette difficulté de dater des fortifications non fouillées subsiste encore aujourd'hui, et justifie déjà qu'on les analyse sur une fourchette chronologique très large. Les communications de Strasbourg ont montré de plus I'intérêt d'une telle démarche, aussi bien pour comparer des formes architecturales permanentes, que pour analyser leur insertion dans le tissu de l'occupation du territoire. Je voudrais ici rappeler quelques expériences que j'ai pu mener sur ce sujet, sans les développer car elles sont en général publiées, et souligner les potentialités des nouveaux outils que les chercheurs ont désormais à leur disposition.

\section{CENT ANS DE RECHERCHE SUR LES ENCEINTES}

La répartition à l'échelle de la France du nombre de fortifications en terre inventoriées par département n'était pas homogène au début $d u x{ }^{e}$ siècle. La liste des enceintes d'A. de Mortillet [1], réalisée juste avant le commencement de l'enquête de la SPF, montrait une densité assez forte en Picardie, Haute-Normandie, Bretagne, Bourgogne, Berry, Poitou, Landes et sur la côte Provençale. Le bilan de I'enquête de la SPF achevé vingt ans après complète la répartition précédente dans le SudOuest, sans vraiment la modifier. C'est peut-être le choix qu'ont fait les enquêteurs d'intégrer les petites enceintes, particulièrement les mottes féodales, qui explique cette répartition inégale, et néanmoins presque identique, sur une période de recherche assez longue et active [2].

À l'échelle de la France septentrionale (au nord d'une ligne Bordeaux-Lyon), et pour le seul âge du Fer, l'enquête que nous avions réalisée au début des années 1980 montre une évolution tout à fait aléatoire de la recherche entre 1876 et 1980 [3]. Jusqu'à 1940, la plupart des « habitats » étudiés sont fortifiés, les habitats isolés ouverts restent très mal connus. À partir de 1980 les prospections et les sauvetages renversent complètement la tendance, et les sites fortifiés non seulement tombent dans I'oubli, sauf quelques rares sites majeurs, mais sont grignotés insidieusement par des constructions diverses sans intervention archéologique. À part au début du $x x^{\mathrm{e}} \mathrm{s}$., période de pleine activité de la Commission des Enceintes, les connaissances progressent suivant des initiatives locales, comme celle de $M$. Wheeler en Bretagne et Normandie [4], ou d'Armand Viré dans le Lot [5].

\section{UNE ÉVOLUTION EXCEPTIONNELLE DES OUTILS : DE LA BOUSSOLE À GOOGLE EARTH}

Les enquêtes sur le terrain, quand on dispose seulement des cartes d'État-Major, du cadastre, des couvertures aériennes verticales de I'I.G.N., et des indications topographiques imprécises de la bibliographie, n'aboutissent qu'après de longs détours dans les fourrés ou les bois et avec un peu de chance. Même si on disposait déjà des couvertures aériennes verticales de I'Institut Géographique National et de stéréoscopes sur le terrain pour voir le relief, la végétation le plus souvent masquait le tracé précis des enceintes. C'est après avoir vécu la difficulté de ces redécouvertes que j'ai d'ailleurs rédigé une thèse sur les problèmes des cartes archéologiques, aux grandes comme aux petites échelles [6], et monté avec plusieurs collègues un projet de base de données pour la France [7] qui a été repris par la Sous-Direction de l'Archéologie.

Avec les outils dont on disposait dans les années 1960, la localisation et le relevé des enceintes faisaient intervenir des méthodes traditionnelles efficaces, -boussole, croquis Gilwell, filicompteur de I'IGN, alidade...) -, mais longues à réaliser et souvent imprécises. Aujourd'hui la mise à disposition sur un simple téléphone des images satellites facilite bien sûr le travail. Géoportail en outre donne accès à de nombreuses cartes, topographiques ou thématiques, à la plupart des couvertures photographiques aériennes verticales, et à des outils de mesure qui permettent une première approximation de la surface couverte par les enceintes. Il faut remarquer toutefois qu'on perd la vision stéréoscopique du relief, alors que cette variable

[1] Mortillet 1906.

[2] BuCHSENSCHUTZ 1971 ; BuChSENSCHUTZ 1984.

[3] BUCHSENSCHUTZ 1984.

[4] WheELER 1957.

[5] VIRÉ 1908.

[6] BUCHSENSCHUTZ 1972.

[7] Buchsenschutz \& Mennessier 1974. 
est essentielle pour interpréter une photographie aérienne : je ne peux pas en faire la démonstration ici, mais tous ceux qui ont participé à des stages de photointerprétation en ont été je crois convaincus. La vision oblique qu'offre aujourd'hui Google Earth, plus riche par certains aspects, ne la remplace pas vraiment parce que I'opérateur ne maîtrise pas les déformations et qu'il n'a jamais une vision globale et stable du site en relief. Toutefois I'imprécision du recouvrement des différentes sources cartographiques dans Géoportail - de I'ordre de plusieurs mètres -, nous a conduits à créer un outil pour réunir sur un SIG des plans de site intégrant à grande échelle les fonds de cartes anciens, les couvertures aériennes et géophysiques, les données de prospection et de fouille [8], à l'image des recherches menées à Meunet-Planches par exemple (fig. 1).

\section{IMAGES, RELEVÉS ET CARTES}

L'outil le plus spectaculaire pour les recherches sur les enceintes de hauteur est bien sûr le Lidar qui est désormais bien connu de tous. Ses avantages sont évidents. Maintenant que son utilité a été largement démontrée, il faut, comme pour les images de satellites ou les supports cartographiques numérisés il y a quelques dizaines d'années, attendre que les services spécialisés mettent à disposition gratuitement ces documents, plutôt que de monter des projets très onéreux pour nos laboratoires. Cela suppose des sacrifices, car dans ces conditions il faut prévoir des projets dans des zones où ces outils sont déjà disponibles, au détriment peut-être des options scientifiques.

Je voudrais attirer aussi l'attention sur une différence fondamentale entre le Lidar et les images satellites redressées, d'une part, les relevés et les cartes d'autre part. Sur le mont Beuvray par exemple, le Lidar a été livré après les relevés au sol faits par une équipe topographique allemande animée par Frantz Schubert [9]. Les deux documents ne sont pas redondants, mais complémentaires : le Lidar donne une image précise du relief, mais elle n'est pas interprétée. Le relevé des topographes guidés par un archéologue identifie, ou même éventuellement accentue ou distingue dans le rendu, les reliefs qui ont de fortes chances d'être d'origine anthropique. C'est ainsi que les voies d'accès et le rempart externe ont pu être identifiés, ce qui n'aurait peut-être pas été possible avec le seul document Lidar.

Dans le même ordre d'idées, il faut attirer l'attention des étudiants sur la différence entre les images satellites diffusées par Google, qui restent des images brutes, et les cartes qui trient, interprètent, et mettent en évidence selon les lois de la sémiologie graphique les phénomènes que I'on veut faire ressortir [10].

Pour la reconnaissance rapide de sites sans mettre en œuvre un appareillage topographique, on peut utiliser une méthode de « relevés expédiés »inspirée des relevés Gilwell, qui privilégient l'interprétation et permettent d'avoir déjà une vision de la problématique du site, avant de mettre en œuvre des relevés topographiques précis. Nous citerons l'exemple du Mont Sainte-Odile, où nous avions commencé ce travail dans l'attente d'un relevé précis [11] (fig. 2).

\section{DÉFENSES, CLÔTURES, PROPRIÉTÉS ET LIEUX CONSACRÉS}

Les sites clôturés dont nous conservons les vestiges en relief constituent une catégorie bien particulière de gisements archéologiques, toutes périodes confondues, comme I'a bien montré ce colloque. La difficulté de leur datation et de leur interprétation a pourtant détourné la plupart des chercheurs de ce thème, qui demandait de forts investissements sur le terrain pour des résultats pas toujours substantiels. Le développement des nouveaux outils permet non seulement d'accélérer la durée des recherches sur le terrain, mais aussi et surtout de publier facilement un état des lieux, comprenant la localisation, une partie de I'histoire du site, l'état de conservation, autant d'éléments qui permettront d'établir une typologie et de piloter des sondages bien ciblés pour dater l'occupation et contrôler la nature des talus.

La prise de conscience de la nature et de I'ancienneté de ces enceintes a été longue, et reste encore sans doute brumeuse dans l'esprit du public. J'en donnerais pour preuve la grande enceinte du Mont Sainte-Odile, dont la datation au haut Moyen Âge a été confirmée à la fois par les fouilles et les datations des tenons en bois il y a plus de vingt ans, mais qui n'est pas encore actée dans les ouvrages de vulgarisation. L'exemple des «Viereckschanzen » est lui aussi symptomatique, et je me sens un peu coupable d'avoir introduit cette discussion en France dans les années 1975. Nous avons en effet défendu au colloque de I'AFEAF de Châteaudun I'interprétation de ces gisements comme des sanctuaires, même si des fermes clôturées protohistoriques en

[8] Chronocarto, Aoroc, Paris ;

http://www.chronocarto.eu/gcserver.

[9] SCHUBERT 1999.

[10] BERTIN 1968.

[11] FICHTL 1994. 

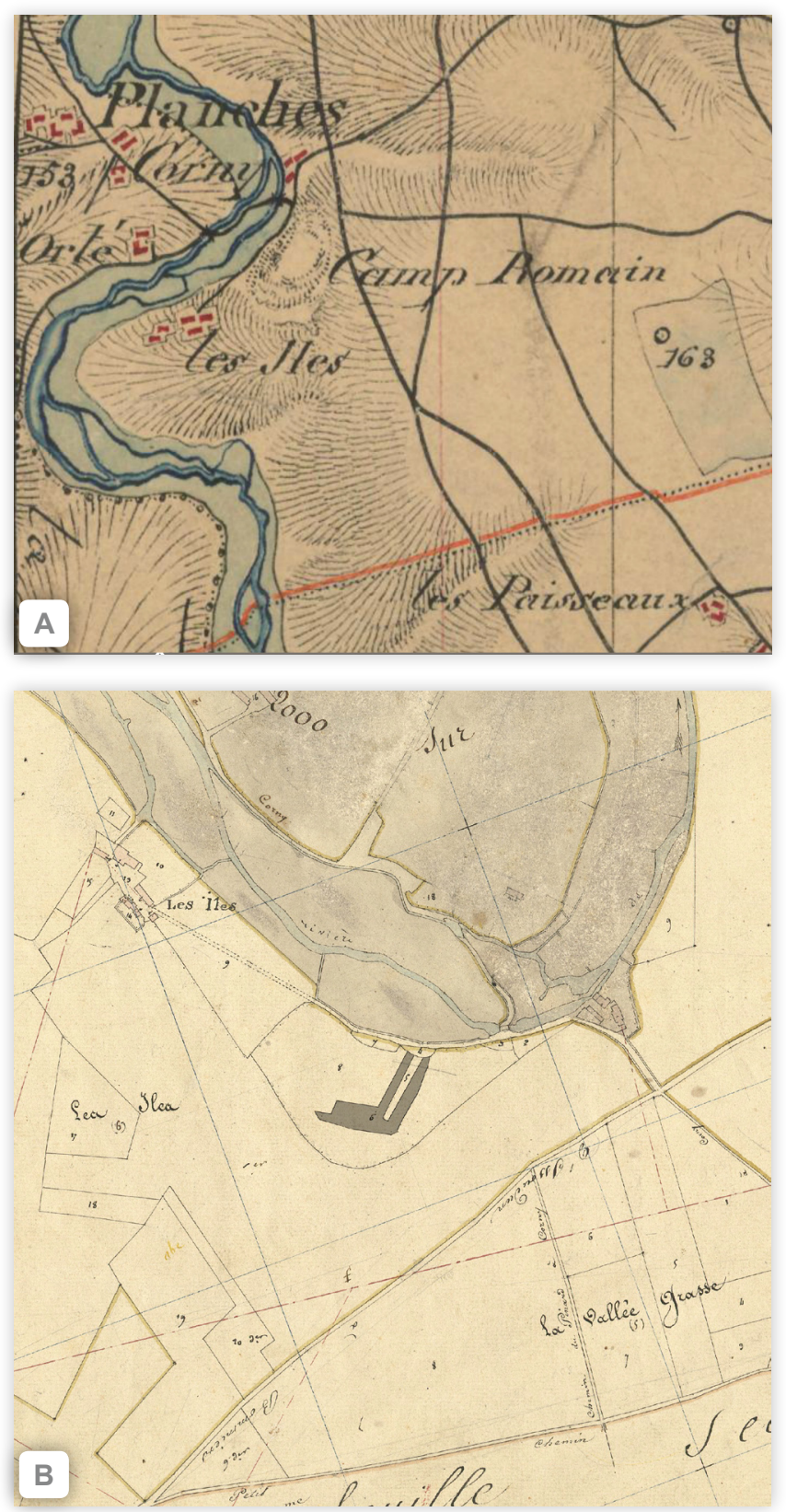
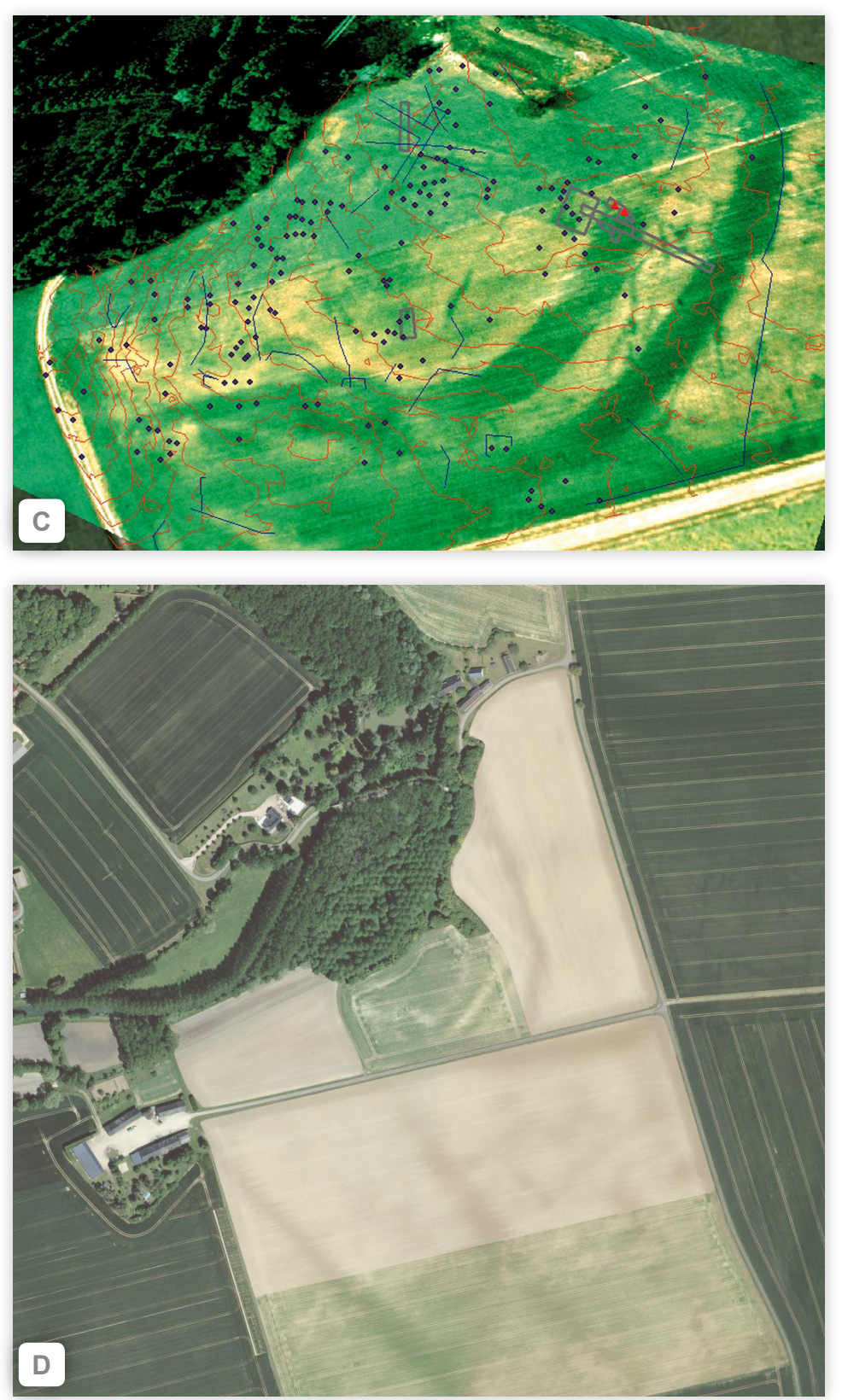

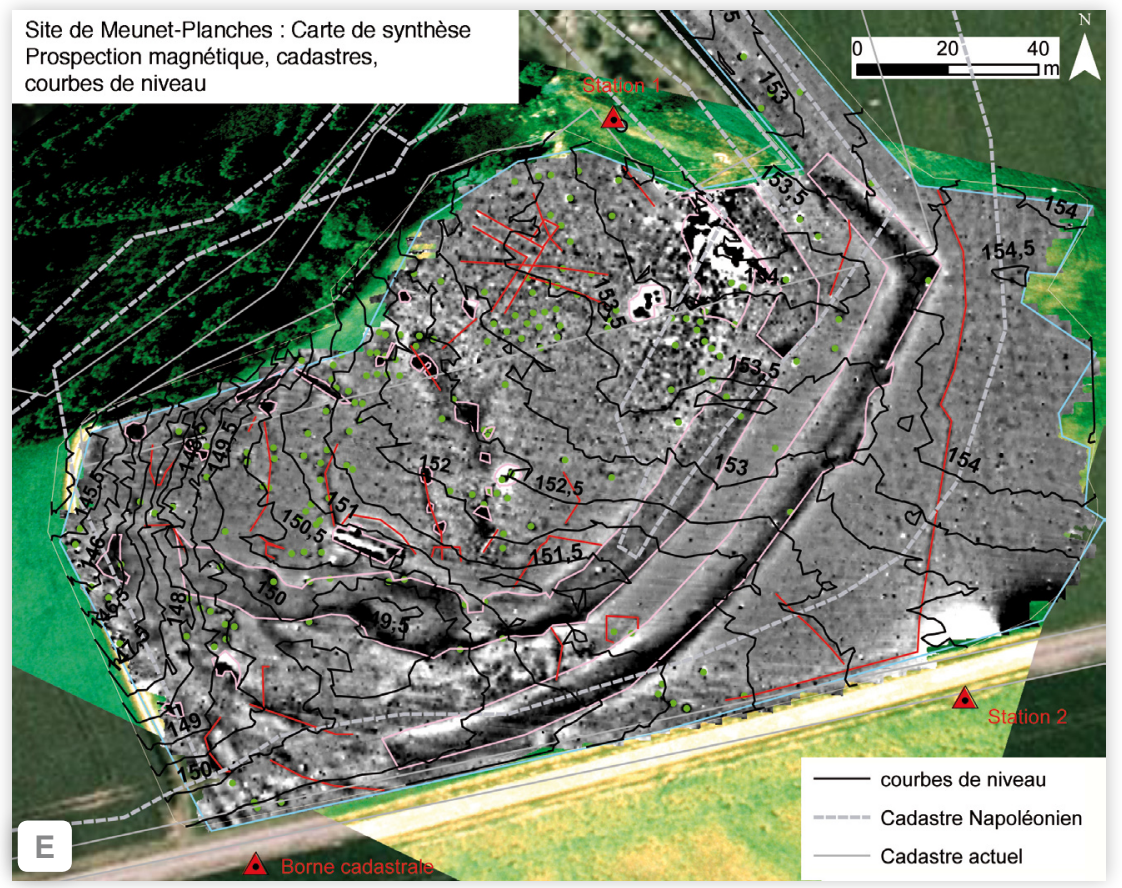

Figure 1

Documentation relative au site de Meunet-Planches :

A : extrait de la carte d'État Major à 1/80 000 .

B : extrait du cadastre napoléonien (l'emplacement du site est coloré).

C : prospection magnétique

emplacement des anomalies et des sondages sur fond de photo aérienne (Jalmain 1976).

D : vue aérienne de 2015 (données IGN).

$E$ : carte de synthèse : prospection magnétique, cadastres, courbes de niveau. 
plaine étaient déjà repérées par les prospections aériennes [12]. On pourra retrouver dans ce colloque I'histoire des recherches, développées surtout en Allemagne, depuis les années 1900. En fait il me semble que le principal obstacle à leur interprétation était dans les a priori mentaux des prospecteurs, moi le premier quand je les ai découverts sur le terrain en 1968 : on ne pouvait pas imaginer à cette époque que des enclos conservés en relief avaient pu subsister depuis la Protohistoire jusqu'à aujourd'hui en relief, malgré les modifications du paysage que nos régions ont connues depuis quelque deux mille ans, ni même que les Celtes avaient pu dessiner des enclos quadrangulaires réguliers pour entourer de simples fermes.

Il est difficile d'étudier des structures dont la fonction nous échappe, surtout quand elles ne sont pas datées. Les suggestions «du bon sens » ne sont pas très fiables parce qu'elles ignorent la plupart des possibilités exploitées au moins pour les sociétés de la Protohistoire. La défense, qu'elle concerne les denrées ou les hommes, vient à l'esprit en premier. Mais quelle défense, pour combien d'agresseurs et de défenseurs, pour résister à des maraudeurs, au coup de main d'une petite compagnie, ou à un long siège en règle ? Les grandes à très grandes surfaces encloses à l'âge du Fer ne sont pas adaptées à la défense, même si pour des raisons de prestige ou de contrôle des entréeset des sorties de biens elles les développent inconsidérément, par exemple au $\mathrm{II}^{\mathrm{e}}$ s. av. J.-C. Les petits sites de hauteur fortifiés de La Tène moyenne et parfois finale, particulièrement en Europe centrale, n'ont pas abrité des habitats, mais des dépôts d'objets métalliques que les détecteurs à métaux ont révélés au cours des dernières décennies: cette destination de simple « lieu consacré » avait jusque-là échappé aux chercheurs.

Les sites de hauteur privilégient en général un rempart imposant, mais dans certains cas ils pouvaient aussi être délimités par des palissades, plus fréquentes dans les fermes de plaine. Il y a en effet des points communs et des types mixtes entre les habitats fortifiés de hauteur et les « résidences aristocratiques » de plaine. Cellesci peuvent êtes délimitées par des clôtures multiples, comme en Grande-Bretagne ou en Bavière au premier âge du Fer. La clôture en soi peut assumer un peu la défense, beaucoup le prestige, l'affirmation de la propriété et la mise en réserve du bois et des chasses. On en trouve la trace depuis les descriptions de Varron jusqu'aux parcs entourés de murs de la période moderne [13].

[12] BuChsenschutz \& OLIVIER 1989.

[13] Varron, De re rustica, III, XII.

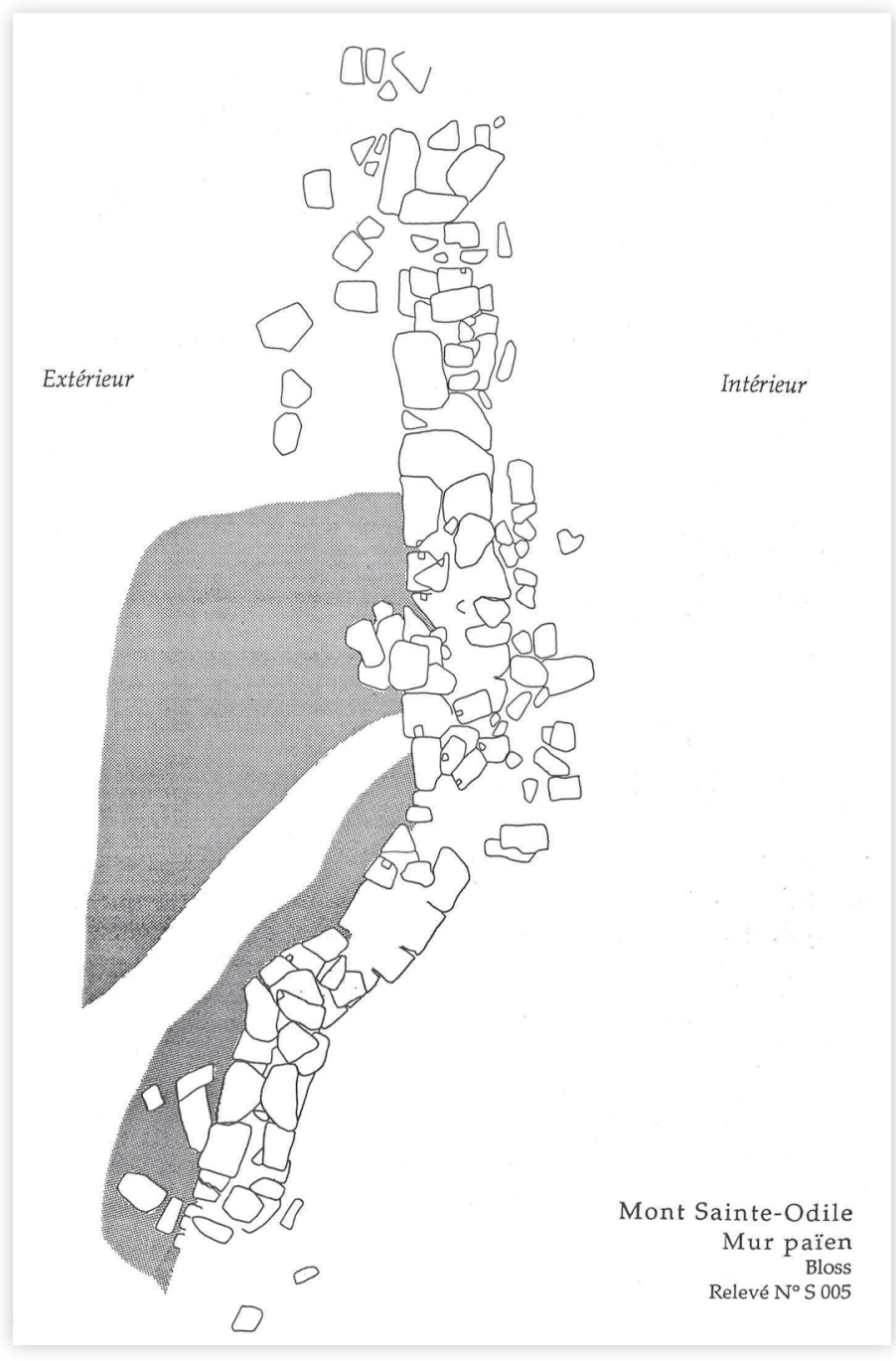

Figure 2

Relevé d'un tronçon du « Mur païen » du Mont Sainte-Odile (d'après FICHTL 1994).

\section{APPLICATION}

Les obstacles matériels à l'étude des enceintes sont aujourd'hui presque tous levés. Organiser des enquêtes systématiques est une affaire de volonté, dès lors que la reconnaissance, I'accès, et les outils de publication sont devenus très faciles à manipuler. On sait que la seule réalisation d'un atlas récompensera déjà cet effort. Mais ce travail sera d'autant plus rentable qu'on n'exclura aucune des périodes - même pas peut-être la ligne Maginot - du corpus. En effet la répartition et les surfaces cumulées des enceintes comparées au reste des terroirs n'auront de sens que si on peut comparer ces chiffres à ceux d'autres périodes. Ce programme ne relève pas uniquement de la recherche de lieux de promenade ou de la manie de la collection. Il peut contribuer à une meilleure compréhension de I'organisation sociale, et particulièrement à l'expression du pouvoir, qu'il s'agisse d'un riche propriétaire, d'un seigneur, ou d'une collectivité toute entière. 
Adam, Anne-Marie, Buchsenschutz, Olivier, Fichtl, Stephan \& Lafon, Xavier, 1994, Le mur païen du mont Sainte-Odile, Ottrott, campagne de fouille 1994, rapport de fouille, Strasbourg, SRA Alsace.

Bertin, Jacques, 1968, Sémiologie graphique, Paris.

Buchsenschutz, Olivier, 1971, «État de la recherche sur les oppida en France, particulièrement dans le Centre », Keltische Oppida in Mitteleuropa und im Karpatenbecken, Symposium, Praha - Liblice, 1970, II. Teil (Archeologické rozhledy 23, 4), Praha, p. 406-416.

Buchsenschutz, Olivier, 1972, Problèmes de cartographie archéologique, thèse de 3ème cycle, université de Paris 1, dactylographiée.

BuchSENSChUtz, Olivier, 1978, « Faux camps romains... vraies enceintes cultuelles? », Travaux militaires en Gaule romaine et dans les provinces du Nord-Ouest, colloque, Paris, ENS, 5-6-7 mai 1978 (Caesarodunum, Supplément 28), p. 287-298.

Buchsenschutz, Olivier, 1984, « 150 ans de recherches sur les fortifications en terre en Europe tempérée », dans A. CahenDelhaye, A. Duval, G. Leman-Delerive \& P. Leman (éd.), Les Celtes en Belgique et dans le nord de la France : les fortifications de l'Âge du Fer, VIe colloque de I'Association française d'étude de l'Âge du Fer, Bavay - Mons, 1982 (Revue du Nord h.s. coll. Archéologie 1) Villeneuve-d'Ascq.

Buchsenschutz, Olivier, 1984, Structures d'habitat et fortifications en France septentrionale, Paris (Mémoires de la Société préhistorique française, 18).

Buchsenschutz, Olivier, 2013, «Citadelles celtiques : défense, prestige et opportunisme », dans Olivier Buchsenschutz, Olivier Dutour \& Claude Mordant (dir.), Archéologie de la violence et de la guerre dans les sociétés pré et protohistoriques, Actes du 136 Congrès National des sociétés historiques et scientifiques, Perpignan (Actes des congrès nationaux des sociétés historiques et scientifiques, éd. électronique).

Buchsenschutz, Olivier \& Mennessier-Jouannet, Christine, 1974, « Projet d'exploitation automatique d'une carte archéologique de la France », dans Mario Borillo \& Jean-Claude Gardin (dir.), Banques de données archéologiques, Colloque Marseille, 12-14 juin 1972, Paris (Colloques nationaux du Centre national de la recherche scientifique, 932), p. 301-312.

Buchsenschutz, Olivier \& Olivier, Laurent (dir.), 1989, Les «Viereckschanzen » et les enceintes quadrilatérales en Europe celtique, IXe colloque de I'AFEAF, Châteaudun, 1985, Paris (Archéologie aujourd'hui, Protohistoire 9).

Buchsenschutz, Olivier, Guillaumet, Jean-Paul \& Ralston, Ian (dir.), 1999, Les remparts de Bibracte : recherches récentes sur la Porte du Rebout et le tracé des fortifications, Glux-en-Glenne (Bibracte 3).

Mortillet, Adrien de, 1906, « Camps et enceintes de France », L'Homme préhistorique 4, p. 193-206.

NicolaI, Caroline von \& Buchsenschutz, Olivier, 1989, « Dépôts métalliques et fortifications de l'âge du Fer européen », dans Sandrine Bonnardin, Caroline Hamon, Michel Lauwers \& Bénédicte Quilliec (dir.), Du matériel au spirituel, Réalités archéologiques et historiques des « dépôts » de la Préhistoire à nos jours, Antibes, p. 321-331.

VIRÉ, Armand, 1908, Inventaire des camps et enceintes du département du Lot, Le Mans.

Wheeler, Robert Eric Mortimer \& Richardson, Katherine M., 1957, Hill-Forts of Northern France, Oxford (Reports of the Research Committee of the Society of Antiquaries of London, 19). 